\title{
Are Northern Irish adolescents meeting physical activity recommendations?
}

\author{
T. A. McCrorie ${ }^{1}$, K. L. Rennie ${ }^{2}$, J. M. W. Wallace ${ }^{1}$ and M. B. E. Livingstone ${ }^{1}$ \\ ${ }^{1}$ Northern Ireland Centre for Food and Health, Biomedical Sciences, University of Ulster, Coleraine BT52 1SA, UK \\ and ${ }^{2}$ Health and Emergency Professions, University of Hatfield, AL10 9AB, UK
}

Current UK physical activity (PA) recommendations for children and adolescents are to achieve a minimum of $60 \mathrm{~min} / \mathrm{d}$ of at least moderate-intensity physical activity ${ }^{(1)}$. Typically, PA levels are determined using activity questionnaires, which are prone to misreporting and are inconsistent in validity and reliability ${ }^{(2)}$. However, data collected using accelerometers provide an objective measure of PA, as well as information on frequency, intensity and time spent in PA.

Adolescents (13-17 years, $n$ 47) from the Coleraine area of Northern Ireland wore a tri-axial accelerometer (RT3, Stay Health Inc, USA) for 7 consecutive days. The time spent in moderate and vigorous PA (MVPA) was determined using cut-offs by Rowland et al. ${ }^{(3)}$. Anthropometric measures and the assessment of body composition by isotope dilution were also performed ${ }^{(4)}$. Body fat was expressed as fat mass index $\left(\mathrm{kg}\right.$ of fat mass $/ \mathrm{m}^{2}$ ) and \% body fat. Lean body mass was also expressed in a similar manner $\left(\mathrm{kg}\right.$ of lean mass $\left./ \mathrm{m}^{2}\right)$. Subjects completed a questionnaire on the time spent in sedentary activity including TV/DVD viewing, internet and computer game use.

Complete accelerometry data ( $\geq 600 \mathrm{~min}$ for a minimum of $3 \mathrm{~d}$, including 1 weekend day) were collected from 41 of the 47 subjects ( 25 boys and 16 girls). Six participants had incomplete data (failure in the field $(n 3)$; monitor malfunction $(n 1)$; non-compliance with the protocol $\left(\begin{array}{l}n \\ 2\end{array}\right)$ ). Ten subjects recorded additional MVPA on one or more days when the RT3 was not worn e.g. watersports or contact sports.

Overall boys were leaner and taller compared to girls $(P<0.05)$. The monitors were worn for a median of $819 \mathrm{~min} / \mathrm{d}(\mathrm{IQR} 783,874)$ on a median of 6 (range 3-7 d) with no significant differences between boys and girls. Boys reported higher levels of sedentary activities $5.25 \mathrm{~h} / \mathrm{d}(3.27,7.57)$ compared to girls $3.25 \mathrm{~h} / \mathrm{d}(2.36,3.82, P<0.05)$. From these measures, $19.5 \%$ of adolescents met the current PA recommendations of $60 \mathrm{~min}$ or more perd of MVPA. Boys with higher MVPA also reported significantly less time spent in sedentary activities $(P<0.05)$. These results also suggest that there was a trend for higher body fat in those adolescents who did not meet the PA recommendations. Conversely, those boys meeting the recommendations had greater lean mass.

In this study, adolescent boys spent more time in MVPA and reported fewer hours spent in sedentary activities compared to adolescent girls. These results demonstrate that only 1 in 5 adolescents are meeting the PA recommendations.

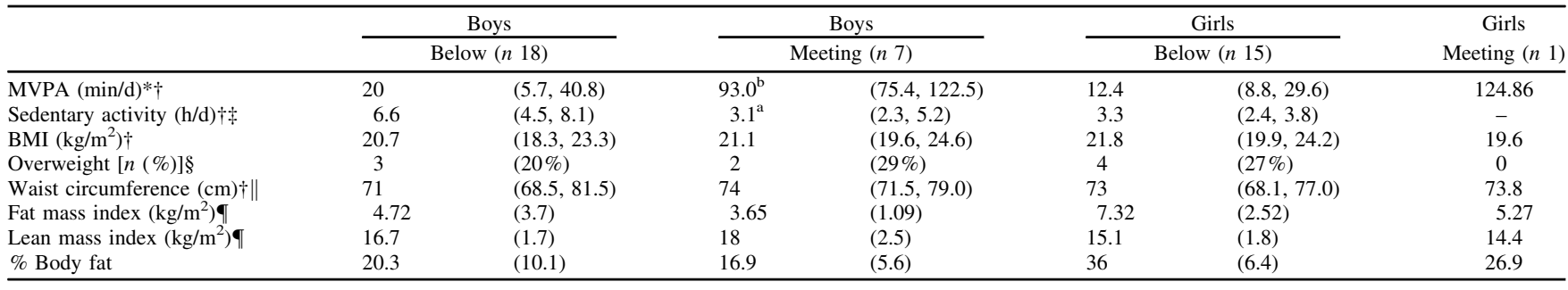

*Time spent in moderate-vigorous intensity activity by objective measure; †median (interquartile range); MVPA group by Mann-Whitney $U$ test; $\neq$ boys $(n$ 23); girls ( $n$ 15);

§overweight and obese determined by IOTF BMI cut-offs ${ }^{(5)}$; $\|$ boys $(n 24)$; girls $(n 15)$; 9 geometric mean $(\mathrm{SE}) ;{ }^{\mathrm{a}} P<0.05$; ${ }^{\mathrm{b}} P<0.001$.

1. Department of Health (2004) At Least Five a Week. Evidence on the Impact of Physical Activity and its Relationship to Health. A Report from the Chief Medical Officer.

2. Livingstone MBE et al. (2003) How active are we? Levels of routine physical activity in children and adults. Proc Nutr Soc. 62, 681-701.

3. Rowlands AV et al. (2004) Validation of the RT3 triaxial accelerometer for the assessment of physical activity. Med Sci Sports Exerc 36, 518-524.

4. McCaffrey TA et al. (2008) Energy density of the diet and change in body fatness from childhood to adolescence; is there a relationship? Am $J$ Clin Nutr 87, 1230-1237.

5. Cole TJ et al. (2000) Establishing a standard definition for child overweight and obesity worldwide: international survey. Br Med J 320, $1240-1243$. 